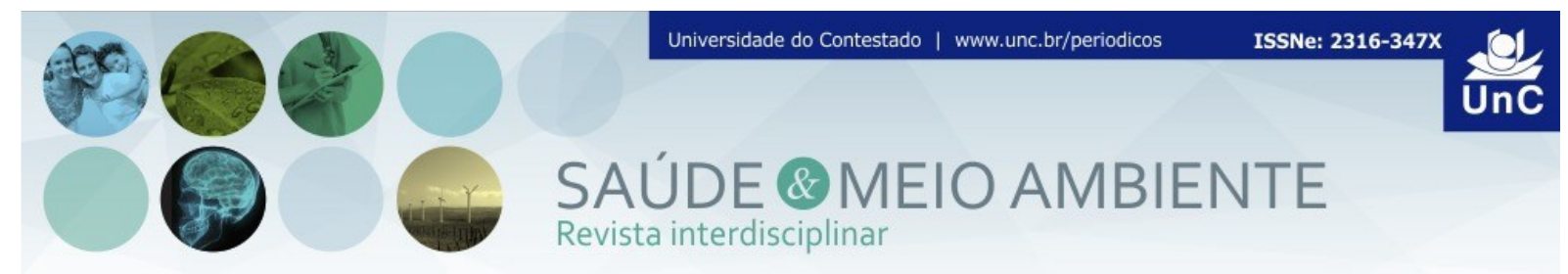

\title{
INTERNAÇÕES HOSPITALARES POR CONDIÇÕES SENSÍVEIS À ATENÇÃO PRIMÁRIA EM IDOSOS DO MEIO OESTE CATARINENSE, ENTRE 2008 A 2015
}

\author{
Bruna da Rocha ${ }^{1}$ \\ Marilia Caroline Lopes Rodrigues ${ }^{2}$ \\ Andreia Dalla Vecchia ${ }^{3}$ \\ Vilma Beltrame 4
}

\begin{abstract}
RESUMO
Introdução: a Lista Brasileira de Internações por Condições Sensíveis à Atenção Primária (ICSAP) é utilizada como instrumento de avaliação do poder de resolutividade da atenção primária e da atenção hospitalar. As ICSAP encontram grande prevalência na população idosa, ocupando grande parte dos leitos hospitalares. Objetivo: analisar a evolução temporal das Internações por Condições Sensíveis à Atenção Primária (ICSAP) em idosos residentes na região da Associação dos Municípios do Meio Oeste Catarinense (AMMOC). Métodos: estudo de análise exploratória e descritiva com abordagem quantitativa de todas internações de idosos por CSAP ocorridas e disponibilizadas do banco de dados do Sistema de Informação Hospitalar do SUS (SIH-SUS) entre 2008 a 2015, na região da AMMOC. Resultados: As principais ICSAP foram doenças pulmonares (1.740) casos, insuficiência cardíaca (1.396) e terceira maior causa foram gastroenterites infecciosas com (861) internações. Houve redução de $21,9 \%$ na taxa de ICSAP durante todo o período analisado. Conclusão: A redução destes índices parece indicar melhorias nos serviços de atenção básica. Este estudo contribuiu para um diagnóstico da real situação, funcionalidade e eficácia dos serviços prestados nos municípios pesquisados.
\end{abstract}

Palavras-chave: Atenção Primária à Saúde. Idosos. Hospitalização. Morbidade.

${ }^{1}$ Graduanda do curso de Enfermagem da Universidade do Oeste de Santa Catarina - UNOESC. Joaçaba, Santa Catarina. Brasil. E-mail: bruna rocha09@hotmail.com

${ }^{2}$ Graduanda do curso de Enfermagem da Universidade do Oeste de Santa Catarina - UNOESC. ID Orcid: Catarina - UNOESC. Joaçaba, Santa Catarina. Brasil. E-mail: srta.maaah@hotmail.com ${ }^{3}$ Bióloga. Bolsista pós-doutoral (PNPD/CAPES) do programa de Mestrado em Biociências e Saúde da Universidade do Oeste de Santa Catarina - UNOESC. Joaçaba, Santa Catarina. Brasil. E-mail: andreiavecchia@gmail.com

${ }_{4}^{4}$ Enfermeira. Doutora em Gerontologia Biomédica. Pesquisadora do programa de Mestrado em Biociências e Saúde e professora do curso de Enfermagem da Universidade do Oeste de Santa Catarina. - UNOESC. Joaçaba, Santa Catarina. Brasil. E-mail: vilma.beltrame@unoesc.edu.br 


\title{
HOSPITAL ADMISSIONS DUE TO CONDITIONS SENSITIVE TO PRIMARY CARE IN THE ELDERLY IN THE MIDDLE OF SANTA CATARINA, BETWEEN 2008 AND 2015
}

\begin{abstract}
Introduction: the Brazilian List of Hospitalizations for Conditions Sensitive to Primary Care (CSAP) is used as an instrument to evaluate the resolving power of primary care and hospital care. The ICSAP found a high prevalence in the elderly population, occupying a large part of the hospital beds. Objective: To analyze the temporal evolution of hospitalizations due to conditions sensitive to primary care (ICSAP) in elderly residents in the region of the Association of Municipalities of Midwest Santa Catarina (AMMOC). Methods: Exploratory and descriptive analysis with a quantitative approach of all hospitalizations of the elderly by CSAP occurred and made available from the SUS Hospital Information System (SIH-SUS) database from 2008 to 2015 in the AMMOC region. Results: The main ICSAP were pulmonary diseases $(1,740)$, heart failure $(1,396)$, and the third largest cause were infectious gastroenteritis with (861) hospitalizations. There was a $21,9 \%$ reduction in the ICSAP rate throughout the analyzed period. Conclusion: The reduction of these indices seems to indicate improvements in basic health care services. This study contributed to a diagnosis of the real situation, functionality and effectiveness of the services provided in the cities surveyed.
\end{abstract}

Keywords: Primary Health Care. Aged. Hospitalization. Morbidity.

\section{INTRODUÇÃO}

A capacidade resolutiva dos serviços de atenção primária na prevenção de hospitalizações desnecessárias tem sido tomada como indicador de qualidade da assistência à saúde ${ }^{1}$. Os indicadores de saúde representam instrumentos essenciais de avaliação e monitoramento dos serviços de saúde ${ }^{2}$. Um destes indicadores, as Internações por Condições Sensíveis à Atenção Primária (ICSAP), correspondem problemas de saúde em estreita relação com os cuidados primários, de modo que serviços de Atenção Primária à Saúde (APS) de boa qualidade podem reduzir risco de internação por essas condições ${ }^{2}$. O índice de ICSAP, inicialmente, foi aplicado nos Estados Unidos onde analisava a acessibilidade da população carente aos serviços de atenção primária. Após, começou ser utilizado para análise dos serviços de forma integral e posteriormente realizados em outros países ${ }^{3}$.

Em algumas condições, a APS oportuna e de qualidade podem evitar e reduzir hospitalizações. Para isto, foi construída uma lista brasileira de ICSAP composta por 19 grupos diagnósticos, a fim de contribuir na avaliação da AP e desempenho dos sistemas de saúde no país ${ }^{4}$.

A construção e análise das taxas de ICSAP podem subsidiar a reorganização da atenção primária no município a partir das desigualdades identificadas, 
contribuindo com planejamentos mais coerentes com as especificidades regionais 5 . Taxas de ICSAP são potencialmente úteis como indicador do impacto da APS, facilitando análise em nível do país, estados e regiões, comparando grupos e municípios com altas ou baixas taxas e características, além de extrema importância no planejamento e gestão dos serviços de saúde ${ }^{6}$. ICSAP representam situações de agravos à saúde que deveriam ser reduzidos na Atenção Básica $(A B)$, evitando grande demanda na atenção terciária ${ }^{3}$.

Sistema de saúde focado no primeiro nível de atenção alcança melhores resultados na saúde. Em situações onde este nível não é resolutivo, ocorrem internações por problemas que possivelmente poderiam ser resolvidos previamente ${ }^{7}$. Acredita-se que hospitalizações desnecessárias refletem falhas do sistema de saúde em não dispor acesso de qualidade à APS, já que o conjunto de doenças responsáveis por tais internações deveriam ser detectadas precocemente ${ }^{2}$.

O acesso ao médico de APS, hospital, nível socioeconômico, organização da atenção primária, critérios para hospitalização e cobertura de seguro de saúde são fatores que influenciam nos índices de ICSAP. A não realização de controle de saúde na ESF implica maior associação com ICSAP e falta de vínculo com a ESF registra probabilidade duas vezes maior de internações por condições sensíveis ao cuidado primário1. Em situações em que a $A B$ não exerce atividades efetivas, associadas com baixa resolutividade ou deficiência na cobertura de serviços, há grande chance de aumento na demanda por internações hospitalares, gerando sobrecarga e despesas devido as ICSAP que seriam evitáveis se cada nível de assistência atuasse de forma correta ${ }^{8}$.

No Brasil, ICSAP foram responsáveis por $28,5 \%$ das internações hospitalares, com taxas de 150 internações por 10.000 habitantes $^{5}$. As internações embora muitas vezes necessárias, podem representar alto risco para a saúde, principalmente na população idosa, que possuem maior risco e vulnerabilidade ao desenvolvimento de doenças ${ }^{9}$.

Neste contexto, objetivou-se analisar as taxas de internação e causas de ICSAP em idosos residentes na região da Associação dos Municípios do Meio Oeste Catarinense (AMMOC) no período de 2008 a 2015, com base em dados do departamento de informática do Sistema Único de Saúde (DATASUS).

\section{METODOLOGIA}

Estudo de análise exploratória e descritiva, com abordagem quantitativa. Informações foram coletadas a partir do banco de dados secundário do Sistema de Informação Hospitalar do SUS (SIH-SUS), do Ministério da Saúde, disponíveis no período de 2008 a $2015^{10}$. Amostra foi composta por todas as internações ocorridas em pessoas com 60 anos ou mais residentes nos municípios que compõem a AMMOC. 
AMMOC é associação jurídica de direito privado, de natureza civil, sem fins lucrativos. Fundada em 1969, tem como sede e foro a cidade e comarca de Joaçaba (SC). É composta pelas pessoas jurídicas de direito público interno e constituída pelos municípios (Água Doce, Capinzal, Catanduvas, Erval Velho, Herval D’Oeste, Ibicaré, Joaçaba, Lacerdópolis, Luzerna, Ouro, Treze Tílias e Vargem Bonita). Sua missão é liderar, planejar, reivindicar e empreender, visando satisfazer as necessidades em âmbito individual e global das administrações municipais, para alcançar os objetivos traçados. Tem por finalidade congregar os municípios associados num fórum permanente de debates acerca das questões comuns das municipalidades e prestar-lhes serviços de natureza técnica especializada, em complementação aos desenvolvidos pelos próprios municípios ${ }^{11}$.

Os dados coletados diretamente no SIH-SUS foram organizados em planilha com as variáveis município de residência, faixa etária e agravos sensíveis por agrupamento. Os agravos pesquisados foram os descritos em Notas Técnicas sobre o Indicador ICSAP ${ }^{12}$ compostos por 19 grupos de causas e 74 diagnósticos classificados de acordo com a décima revisão da Classificação Internacional de Doenças (CID10). Os 18 grupos investigados integram uma gama de diferentes CID e foram classificadas em: Doenças preveníeis por imunização e condições sensíveis; gastroenterites infecciosas e complicações; deficiências nutricionais de vitaminas, sais minerais e proteico calórica; infecções de ouvido, nariz e garganta; pneumonias bacterianas; asma; doenças pulmonares; hipertensão; angina; insuficiência cardíaca; doenças cerebrovasculares; diabetes mellitus com e sem complicações associadas; epilepsias; infecção no rim e trato urinário; infecção da pele e tecido subcutâneo; doença inflamatória órgãos pélvicos femininos e úlcera gastrointestinal. $\mathrm{O} 19^{\circ}$ grupo de agravos não foi inserido no estudo, pois trata-se de internações relacionadas ao pré-natal e parto, grupo que a população não foi exposta ${ }^{13}$.

As informações coletadas foram importadas para tabelas do Excel e posteriormente analisadas através de estatística descritiva. As variáveis foram expressas em número absoluto percentuais, conforme a forma de apresentação. Para o cálculo da taxa de internação (TI) utilizou-se a seguinte fórmula:

$$
T . I=\frac{\text { Número de internações }}{\text { População residente }} \times 1000
$$

A população residente de cada município foi extraída do site do DATASUS (http://tabnet.datasus.gov.br/cgi/tabcgi.exe?ibge/cnv/poptsc.def.) com base no IBGEEstimativas de população.

Todos os preceitos contidos na resolução CNS-466/2012 foram seguidos. Por se tratar de pesquisa em banco de dados secundários e disponível a visitação pública, não foi necessário o Termo de Consentimento Livre e Esclarecido. Este estudo não ofereceu risco, uma vez que não houve abordagem direta com os participantes e não houve possibilidade de identificação. Estudo aprovado pelo Comitê de Ética e Pesquisa (CEP) da Universidade do Oeste de Santa Catarina (UNOESC) sob parecer $n^{\circ} .2 .609 .865$. 


\section{RESULTADOS}

O estudo sobre as taxas de internações em idosos por condições sensíveis a $A B$ da região da AMMOC registrou 7.485 ICSAP nos municípios pesquisados, entre 2008 a 2015. No geral, dados apontaram redução das taxas de internação durante todo período do estudo, cinco municípios (Água doce, Erval Velho, Ibicaré, Luzerna e Vargem Bonita) apresentaram aumento nas taxas de ICSAP no transcorrer de 2008 a 2015 (tabela 1). Demais municípios apresentam redução das taxas de internações em relação ao ano de 2008. Salienta-se aumento contínuo nas taxas de internação para Água doce desde 2010, em relação ao ano inicial, onde passou de 10,6 em 2008 para 29,4 internações em 2014.

Tabela 1 - Taxa de Internação Hospitalar por ICSAP em idosos residentes nos municípios do Meio Oeste Catarinense nos anos de 2008 a 2015.

\begin{tabular}{lrrrrrrrrr}
\hline Município/taxa* $^{*}$ & $\mathbf{2 0 0 8}$ & $\mathbf{2 0 0 9}$ & $\mathbf{2 0 1 0}$ & $\mathbf{2 0 1 1}$ & $\mathbf{2 0 1 2}$ & $\mathbf{2 0 1 3}$ & $\mathbf{2 0 1 4}$ & $\mathbf{2 0 1 5}$ & Média \\
\hline Água Doce & 10,6 & 8,4 & $\mathbf{1 2 , 2}$ & $\mathbf{1 6 , 0}$ & $\mathbf{1 4 , 9}$ & $\mathbf{1 4 , 7}$ & $\mathbf{2 9 , 4}$ & $\mathbf{1 5 , 0}$ & 15,1 \\
Capinzal & 11,2 & 11,1 & 9,9 & 5,6 & 7,4 & 8,7 & 6,8 & 4,5 & 8,1 \\
Catanduvas & 6,9 & 6,6 & 4,7 & 4,7 & 6,5 & 5,3 & 4,1 & 5,3 & 5,51 \\
Erval Velho & 7,8 & 7,3 & 7,1 & 6,6 & 8,0 & 7,4 & $\mathbf{1 1 , 2}$ & $\mathbf{1 0 , 3}$ & 6,9 \\
Herval d'Oeste & 10,3 & 7,6 & 7,4 & 6,7 & 7,4 & 6,3 & 7,7 & 5,2 & 7,3 \\
Ibicaré & 6,6 & $\mathbf{1 0 , 1}$ & $\mathbf{9 , 7}$ & 5,3 & $\mathbf{1 1 , 0}$ & $\mathbf{9 , 2}$ & $\mathbf{1 4 , 0}$ & $\mathbf{7 , 8}$ & 8,4 \\
Joaçaba & 8,2 & 6,7 & 6,1 & 6,4 & 6,6 & 6,5 & 6,8 & 3,6 & 6,3 \\
Lacerdópolis & 6,6 & 7,5 & 2,2 & 5,9 & 5,9 & 4,9 & 4,4 & 5,7 & 5,4 \\
Luzerna & 12,1 & 8,1 & 10,5 & 8,5 & 9,8 & $\mathbf{1 2 , 2}$ & $\mathbf{1 5 , 7}$ & $\mathbf{1 3 , 5}$ & 11,3 \\
Ouro & 7,8 & 9,9 & 6,9 & 7,3 & 9,5 & 8,4 & 9,4 & 5,1 & 8,0 \\
Treze Tílias & 12,3 & 12,4 & 11,1 & 8,6 & 5,1 & 4,4 & 2,4 & 3,1 & 7,4 \\
Vargem Bonita & 4,3 & $\mathbf{4 , 9}$ & $\mathbf{6 , 6}$ & $\mathbf{7 , 3}$ & $\mathbf{5 , 9}$ & $\mathbf{1 0 , 7}$ & $\mathbf{6 , 5}$ & 2,5 & 6,0 \\
\hline
\end{tabular}

Fonte: Ministério da Saúde/Sistema de Informações Morbidade Hospitalar do SUS (SIH/SUS) ${ }^{10}$ e base demográfica do IBGE.

* Taxa por 1.000 habitantes.

Na figura 1 descrevemos a média das taxas de ICSAP na população de idosos residentes na região da AMMOC em cada ano pesquisado. Ao analisarmos a média geral por ano de todos municípios estudados, contatou-se diminuição temporal das taxas de ICSAB, tendo em 2008 taxa de 8,7 internações por 1.000 habitantes e no último ano, em 2015 6,8 internações por 1.000 habitantes. 
Figura 1 - Média anual das taxas de Internação Hospitalar por ICSAP em idosos residentes nos municípios da AMMOC, no período de 2008 a 2015.

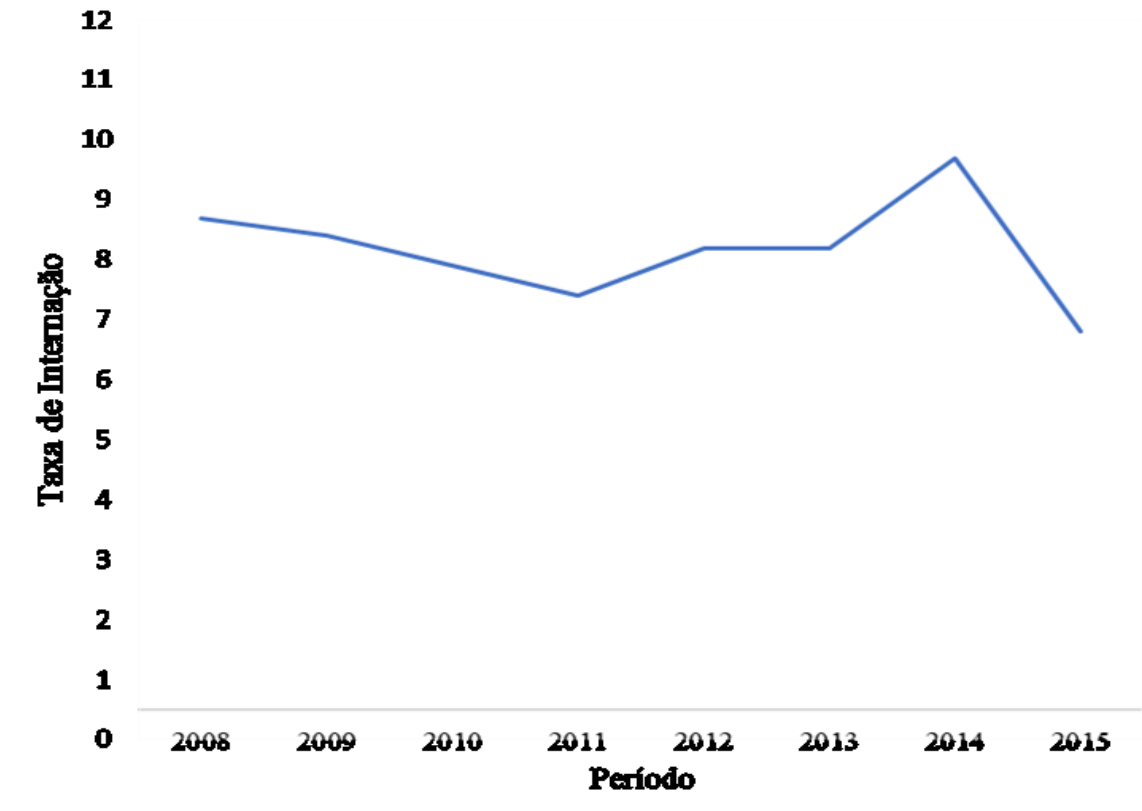

Na tabela 2 descrevemos a quantidade total e média de ocorrência por ano de ICSAP para a população de idosos, no período de 2008 a 2015 em cada município da AMMOC, apresentando e destacando as principais causas de internações. 
Tabela 2. Internações por Condições Sensíveis à Atenção Primária - ICSAP em idosos residentes nos municípios da AMMOC, no período de 2008 a 2015.

\begin{tabular}{|c|c|c|c|c|c|c|c|c|c|c|c|c|c|}
\hline Internações /CSAP & $\begin{array}{l}\text { Água } \\
\text { doce } \\
t(m)^{*}\end{array}$ & $\begin{array}{c}\text { Capinzal } \\
t(m)^{*}\end{array}$ & $\begin{array}{l}\text { Catanduva } \\
t(m)^{*}\end{array}$ & $\begin{array}{l}\text { Erval } \\
\text { Velho } \\
t(m)^{*}\end{array}$ & $\begin{array}{c}\text { Herval } \\
\text { d'Oeste } \\
t(m)^{\star}\end{array}$ & $\begin{array}{c}\text { Ibicaré } \\
t(m)^{*}\end{array}$ & $\begin{array}{c}\text { Joaçaba } \\
t(m)^{*}\end{array}$ & $\begin{array}{l}\text { Lacerdópolist } \\
\qquad(\mathrm{m})^{*}\end{array}$ & $\begin{array}{l}\text { Luzerna } \\
t(m)^{*}\end{array}$ & $\begin{array}{l}\text { Ouro } \\
t(m)^{*}\end{array}$ & $\begin{array}{l}\text { Treze } \\
\text { Tílias } \\
\mathbf{t}(\mathrm{m})^{*}\end{array}$ & $\begin{array}{c}\text { Vargem } \\
\text { Bonita } \\
(\mathrm{m})^{*}\end{array}$ & Total \\
\hline $\begin{array}{l}\text { Doenças preveníveis } \\
\text { p/imuniz/condições sensiveis } \\
\text { Gastroenterites infecciosas e } \\
\text { complicações }\end{array}$ & $\begin{array}{c}2(0,3) \\
158 \\
(19,8)\end{array}$ & $\begin{array}{c}3(0,4) \\
216(27)\end{array}$ & $\begin{array}{c}0 \\
40(5)\end{array}$ & $\begin{array}{c}0 \\
48(6)\end{array}$ & $\begin{array}{c}2(0,3) \\
85(10,6)\end{array}$ & $\begin{array}{c}0 \\
16(2)\end{array}$ & $\begin{array}{c}0 \\
79(9,9)\end{array}$ & $\begin{array}{c}0 \\
4(0,5)\end{array}$ & $\begin{array}{c}0 \\
64(8)\end{array}$ & $\begin{array}{c}1(0,1) \\
89(11,1)\end{array}$ & $\begin{array}{c}0 \\
43(5,4)\end{array}$ & $\begin{array}{c}0 \\
19(2,4)\end{array}$ & $\begin{array}{c}8 \\
861\end{array}$ \\
\hline Anemia & $7(0,9)$ & $15(1,9)$ & $1(0,1)$ & $1(0,1)$ & $29(3,6)$ & $10(1,3)$ & $27(3,4)$ & $8(1)$ & $32(4)$ & $6(0,8)$ & $1(0,1)$ & 0 & 137 \\
\hline Deficiências nutricionais & $37(4,6)$ & 0 & $4(0,5)$ & $3(0,4)$ & $4(0,5)$ & $5(0,6)$ & $4(0,5)$ & 0 & $9(1,1)$ & 0 & 0 & $10(1,3)$ & 76 \\
\hline Pneumonias bacterianas & $82(10,3)$ & $83(10,4)$ & $42(5,3)$ & $7(0,9)$ & $57(7,1)$ & $15(1,9)$ & $72(9)$ & $2(0,3)$ & $34(4,3)$ & $31(3,9)$ & $38(4,8)$ & $9(1,1)$ & 472 \\
\hline Asma & $50(6,3)$ & $15(1,9)$ & $3(0,4)$ & $6(0,8)$ & $8(1)$ & 0 & $12(1,5)$ & 0 & $20(2,5)$ & $15(1,9)$ & $8(1)$ & $1(0,1)$ & 138 \\
\hline Doenças pulmonares & $176(22)$ & $382(47,8)$ & $102(12,8)$ & $86(10,8)$ & $285(35,6)$ & $67(8,4)$ & $\begin{array}{c}341 \\
(42,6)\end{array}$ & $30(3,8)$ & $68(8,5)$ & $74(9,3)$ & $90(11,3)$ & $39(4,9)$ & 1740 \\
\hline Hipertensão & $40(5)$ & $71(8,9)$ & $28(3,5)$ & $13(1,6)$ & $29(3,6)$ & $12(1,5)$ & $79(9,9)$ & $12(1,5)$ & $40(5)$ & $29(3,6)$ & $21(2,6)$ & $18(2,3)$ & 392 \\
\hline Angina & $22(2,8)$ & $36(4,5)$ & $19(2,4)$ & $12(1,5)$ & $78(9,8)$ & $22(2,8)$ & $95(11,9)$ & $1(0,1)$ & $27(3,4)$ & $26(3,3)$ & $28(3,5)$ & $13(1,6)$ & 379 \\
\hline Insuficiência cardíaca & $\begin{array}{c}127 \\
(15,9)\end{array}$ & $207(25,9)$ & $82(10,3)$ & $56(7)$ & $303(37,9)$ & $40(5)$ & $\begin{array}{c}291 \\
(36,4)\end{array}$ & $10(1,3)$ & $57(7,1)$ & $77(9,6)$ & $84(10,5)$ & $62(7,8)$ & 1396 \\
\hline Doenças cerebrovasculares & $62(7,8)$ & $101(12,6)$ & $53(6,6)$ & $18(2,3)$ & $176(22)$ & $25(3,1)$ & $\begin{array}{c}183 \\
(22,9)\end{array}$ & $12(1,5)$ & $60(7,5)$ & $36(4,5)$ & $26(3,3)$ & $31(3,9)$ & 783 \\
\hline Diabetes mellitus & $61(7,6)$ & $87(10,9)$ & $16(2)$ & $6(0,8)$ & $113(14,1)$ & $17(2,1)$ & $120(15)$ & $6(0,8)$ & $42(5,3)$ & $20(2,5)$ & $21(2,6)$ & $14(1,8)$ & 523 \\
\hline Epilepsias & $1(0,1)$ & $3(0,4)$ & $2(0,3)$ & $4(0,5)$ & $4(0,5)$ & 0 & $2(0,3)$ & 0 & $10(1,3)$ & $9(1,1)$ & $2(0,3)$ & $1(0,1)$ & 38 \\
\hline Infecção no rim e trato urinário & $24(3)$ & $81(10,1)$ & $32(4)$ & $22(2,8)$ & $43(5,4)$ & $17(2,1)$ & $65(8,1)$ & $12(1,5)$ & $26(3,3)$ & $41(5,1)$ & $10(1,3)$ & $9(1,1)$ & 382 \\
\hline $\begin{array}{l}\text { Infecção da pele e tecido } \\
\text { subcutâneo } \\
\text { Úlcera gastrointestinal }\end{array}$ & $\begin{array}{l}3(0,4) \\
1(0,1)\end{array}$ & $19(2,4)$ & $7(0,9)$ & $4(0,5)$ & $4(0,5)$ & $\begin{array}{l}1(0,1) \\
3(0,4)\end{array}$ & $6(0,8)$ & 0 & $\begin{array}{l}1(0,1) \\
3(0,4)\end{array}$ & $\begin{array}{l}9(1,1) \\
8(1)\end{array}$ & $2(0,3)$ & $1(0,1)$ & 55 \\
\hline $\begin{array}{l}\text { Infecção do ouvido, nariz e } \\
\text { garganta }\end{array}$ & 0 & $3(0,4)$ & $1(0,1)$ & 0 & 0 & 0 & $1(0,1)$ & 0 & $5(0,6)$ & $4(0,5)$ & $2(0,3)$ & 0 & 16 \\
\hline $\begin{array}{l}\text { Doença Inflamatória órgãos } \\
\text { pélvicos femininos }\end{array}$ & 0 & 0 & $1(0,1)$ & 0 & $1(0,1)$ & 0 & 0 & 0 & 0 & 0 & $4(0,5)$ & 0 & 6 \\
\hline $\begin{array}{l}\text { Total de internações, (média } \\
\text { lano). }\end{array}$ & $\begin{array}{c}853 \\
(106,6)\end{array}$ & 1341 (167) & $432(54)$ & $288(36)$ & 1236 (1545) & $\begin{array}{c}250 \\
(31,3)\end{array}$ & $\begin{array}{l}1393 \\
(174)\end{array}$ & $97(12,1)$ & $511(63,9$ & $475(59,4)$ & $380(47,5)$ & $229(28,6)$ & 7485 \\
\hline
\end{tabular}

${ }^{*}$ t corresponde ao número total de internações por CSAP em cada município e $(\mathrm{m})$ a média de internações por ano ocorridas no período de 2008 a 2015. 


\section{DISCUSSÃO}

Neste estudo, no geral, a maior parte dos munícipios apresentou redução nas taxas de ICSAP ao longo do período. A taxa média em cada município não passou de 15,1 internações para 1.000 habitantes ao ano (tabela 1) e apresentaram valores inferiores as encontradas em outras pesquisas. Estudo realizado entre 2000 e 2010 com base em dados do SIH, SUS e SIAB no estado do Rio de Janeiro analisou a evolução temporal das ICSAP na população acima de 60 anos e constataram em 2000 o grupo de idosos de 70 a 74 anos apresentava taxas de ICSAP em torno de 56 por 1.000 habitantes, enquanto idosos de 60 a 64 anos apresentavam taxa 36 por 1.000. Já em 2010 , as taxas foram de aproximadamente $28 / 1.000$ para a faixa etária de 70 a 74 anos e 18/1.000 para os idosos de 64 a 69 anos $^{9}$. Em Minas Gerais e Rio de Janeiro, autores descrevem redução de internações por causas consideradas evitáveis com atenção primária, no período de 2000 a 2012. A diminuição das taxas de ICSAP foram observadas em todas as idades dentro da faixa etária de 60 a 74 anos $^{8}$. No Paraná, entre 2000 a 2012, houve decréscimo de 30,6\% em internações por CSAP, passando de 59.593 para $41.334^{14}$.

Salientamos que Água Doce apresentou de forma contínua, aumento nas taxas de ICSPA. Este munícipio diferentemente dos demais, possui IDH (Índice de Desenvolvimento Humano) geral de 0,698 pontos, valor considerado médio, no entanto apresenta o menor IDH entre os municípios pesquisados, ocupando a $237^{\circ}$ posição no estado de SC. Entre as cidades com redução das taxas de internações, destaca-se Joaçaba e Treze Tílias que mantiveram índices contínuos desde 2008 até 2015. Joaçaba é maior cidade da região da AMMOC e apresenta a melhor posição no ranking IDH ocupando a $3^{\text {a }}$ posição em SC com 0,827 pontos, considerada alta. Em seguida, também com elevado IDH aparece Treze Tílias com 0,795 pontos e apresentou redução importante nas taxas de internações, passando de 12,3 em 2008 para 3,1 internações ao ano em $2015^{15}$.

É possível traçar a relação entre as taxas de ICSAP e o IDH de cada município se considerar, por exemplo, Joaçaba $\left(3^{\circ}\right)$ e Treze Tílias $\left(14^{\circ}\right)$ que iniciaram o período de pesquisa com taxas maiores de ICSAP, mas ao final do estudo apresentaram quedas das taxas de internação. Luzerna é exceção, pois ainda que apresente o $19^{\circ}$ melhor IDH do estado de SC, teve aumento nas taxas de ICSAP principalmente entre 2013 a 2015 . Já Agua Doce $\left(237^{\circ}\right)$, Ibicaré $\left(207^{\circ}\right)$ e Erval Velho $\left(175^{\circ}\right)$ e Vargem Bonita $\left(185^{\circ}\right.$, embora houve redução em 2015 , teve aumento de internações nos outros 6 anos) estão entre os piores colocados no ranking de IDH catarinense entre os municípios da AMMOC e apresentaram aumento das taxas de ICSAP comparando os anos de 2015 e 2008, podendo estabelecer uma relação entre o aumento e elevadas taxas de internação ao nível de IDH dos municípios. Há também exceção de Catanduvas $\left(198^{\circ}\right)$ que apresentou diminuição das taxas de ICSAP durante o período de 2008 a 2015 embora ocupem posições intermediárias no ranking de IDH. As exceções talvez possam ser explicadas pelo fato de que ainda que a posição ocupada pelo município no ranking do IDH pareçam ser as posições finais, a pontuação obtida não difere muito uma da outra e ainda é considerada alta, já que o estado apresenta um dos IDH mais 
elevados do país, sendo que apenas Água Doce está na faixa intermediária do ranking, ainda considerado índice médio ${ }^{15}$.

Analisando a evolução temporal, analisando as taxas de todos os municípios ano a ano (figura 1), verifica-se que em 2008 obtivemos taxa média de 8,7 internações por 1.000 habitantes, em seguida houve redução a cada ano até 2014, onde houve aumento para o maior índice de todo o período atingindo 9,7 internações por 1.000 habitantes. Novamente em 2015 houve diminuição no menor índice com 6,8 internações por 1.000 habitantes. Com isso, observou-se redução de $21,9 \%$ nas taxas de internação total. Apenas no ano de 2014 verificou-se aumento de internações em relação a 2008 e a redução destes índices parece indicar melhorias no sistema de saúde desta região. Nossos dados apresentaram taxas menores em relação outros achados. Estudos brasileiros indicam que as taxas de ICSAP variaram de 149,6 a 143,3 por 10.000 habitantes $^{16}$. No Rio Grande do Sul foram observadas taxas de 14,5 por 1.000 habitantes ${ }^{17}$, de 10,8 por 1.000 em São Paulo ${ }^{18}$ e em Belo Horizonte, a taxa foi de 154,0 por 10.000 habitantes $^{19}$.

Em se tratando das principais causas associadas a ICSAP, embora houve registro (menor número) em todos os 18 grupos pesquisados, destacaremos as três principais ocorridas na região da AMMOC. Conforme tabela 2, a principal causa de internação foi relacionada as doenças pulmonares com 1.740 casos $(23,24 \%)$, dos quais 9 dos municípios (exceto Herval d' Oeste, Ouro e Vargem Bonita) apresentaram maior índices de internações, corroborando com outros estudos. No estado do Rio de Janeiro, grande parte das ICSAP foram por doenças do aparelho respiratório, especificamente as doenças pulmonares obstrutivas crônicas com mais de $50 \%$ das ICSAP analisadas ${ }^{9}$. Dados semelhantes foram encontrados em grupo com idade superior a 65 anos, onde as doenças pulmonares ocuparam lugar de destaque entre as principais ICSAP ${ }^{20}$. As doenças pulmonares enquadram-se como a quarta causa de morte em indivíduos acima de 70 anos devido ao processo de envelhecimento. Desta forma, os problemas respiratórios se desenvolvem mais facilmente e sua resolubilidade com o avançar da idade fica mais difícil. Os fatores ambientais, estilo de vida e tabagismo têm grande influência na saúde respiratória ${ }^{21}$.

No Brasil, conforme análise de dados do Sistema de Informações sobre Mortalidade, tem mostrado crescimento das internações e óbitos por doenças respiratórias em idosos, mesmo considerando o envelhecimento da população ${ }^{22}$. As vacinas para o sistema respiratório são benéficas aos indivíduos idosos, especialmente para aqueles que têm doenças pulmonares ${ }^{21}$.

Em segundo lugar, a doença mais frequente apontada neste estudo foi insuficiência cardíaca com 1.396 (18,65\%) internações. Observou-se maior ocorrência nos municípios de Capinzal, Herval d' Oeste e Joaçaba (tabela 2). Semelhanças foram encontradas em estudo de Sousa et al. (2016) ${ }^{20}$ que além das doenças pulmonares, a insuficiência cardíaca também ocupa posição de destaque nas ICSAP. Magalhães e Morais Neto $(2017)^{5}$ analisando dados de internação da cidade de Goiânia na faixa etária de 60 anos ou mais, também encontraram nas doenças do aparelho circulatório as condições mais prevalentes, tendo a insuficiência cardíaca e angina como os principais grupos de causa com as maiores taxas de ICSAP. Insuficiência cardíaca também ocupou segundo lugar com 15,5\% 
das internações, na cidade de Cotia-SP ${ }^{7}$. Estudo analisando taxas de ICSAP e principais grupos de causas no gasto com ICSAP de adultos e idosos, observou maior proporção com doenças cardiovasculares, incluindo a insuficiência cardíaca23.

Entre as cardiopatias, a insuficiência cardíaca crônica (ICC) é a principal causa de consulta médica para homens e mulheres acima de 75 e 85 anos, respectivamente ${ }^{24}$. A ICC afeta predominantemente a população idosa com prevalência aproximada de $7 \%$ nos indivíduos com idade entre 75 e 84 anos, e superior a $15 \%$ em idosos com mais de 85 anos $^{25}$.

Pacientes com doenças crônicas como ICC requerem ajustamento individual do tratamento, assistência e adaptações a novos estilos de vida. Neste sentido, a OMS define adesão como sendo o grau em que o comportamento de uma pessoa, em relação a tomar o medicamento, seguir um regime alimentar e executar mudanças no estilo de vida corresponde às recomendações acordadas com um prestador de saúde ${ }^{26}$. Dentre as mudanças necessárias no estilo de vida destaca-se a alteração nutricional com redução de sódio, o controle hídrico, a restrição da ingestão de álcool, a monitorização do peso diário, a vacinação específica e a suspensão do tabagismo ${ }^{27}$.

A terceira condição mais frequente encontrada neste estudo, também de relevância mundial, foram gastroenterites infecciosas e suas complicações, totalizando $861(11,50 \%)$ internações de 2008 a 2015 e esteve presente com maior frequência nos municípios Água Doce, Capinzal e Ouro (tabela 2). As gastroenterites virais são patologias extremamente comuns, sendo a segunda maior causa de doença infecciosa e a maior causa de diarreia infecciosa, estimando em torno de 2,5 milhões de mortes em todo o mundo, afetando todas as idades, porém, mais frequentes em crianças e idosos ${ }^{28}$.

De acordo com OMS, ocorrem cerca de 2 bilhões de casos de gastroenterites infecciosas ao ano, podendo ser parasitária, bacteriana ou viral. No entanto neste estudo, os dados não permitem identificar a origem da infecção. Os principais agentes bacterianos relacionados com gastroenterites incluem os gêneros Salmonella, Shigella, Escherichia, Staphylococcus, Aeromonas, Plesiomonas, Yersinia e Campylobacter. Entre os parasitas, os mais frequentes são Giardia lamblia, Entamoeba histolytica e Cryptosporidium parvum. Os principais agentes etiológicos virais são rotavírus, norovírus, astrovírus e adenovírus. Destaca-se o rotavírus, responsável por $60 \%$ de todos os episódios diarreicos nos países em desenvolvimento e $40 \%$ nos países desenvolvidos. O patógeno é, mundialmente, indicado como causador de $20 \%$ a $70 \%$ das internações hospitalares por diarreias ${ }^{28}$ e a principal causa de gastroenterite aguda em crianças menores de 5 anos, podendo causar cerca de 215 mil óbitos ao ano ${ }^{29}$.

Entre os municípios pesquisados foram registrados queda nas taxas de ICSAP em sete e em cinco houve aumento das taxas (tabela 1). Todos os municípios do estudo implantaram $A B$ na modalidade de PSF/ESF e possuem cobertura de atenção básica a saúde em $100 \%$ da população, exceto Capinzal e Ouro que permanecem com menos de $50 \%$ de cobertura. Mesmo sem cobertura 
total da ESF, Capinzal e Ouro registram queda nas taxas de ICSAP quando avaliamos o ultimo ao primeiro ano da pesquisa. Redução também observadas nos municípios de Catanduvas, Herval d'Oeste, Joaçaba, Lacerdópolis e Treze Tílias que apresentam cobertura total. Por outro lado, apesar de apresentar cobertura de $100 \%$, Água Doce, Erval Velho, Ibicaré, Luzerna e Vargem Bonita registraram aumento nas taxas de internação durante o período pesquisado.

Resultados de estudos sobre ICSAP no Brasil e em outros países mostram que é possível monitorar e avaliar o acesso e a efetividade da atenção primária de um Sistema de Saúde Universal como SUS utilizando indicadores epidemiológicos construídos a partir das ICSAP 5 .

Segundo estudo, há tendência ao declínio das taxas de internações por pneumonia em maiores de 60 anos na população com atenção ambulatorial básica adequada e não adequada. As taxas por diarreia apresentaram tendência à estabilidade. Em relação às internações por infarto agudo do miocárdio, tanto nos municípios com atenção adequada quanto naqueles com atenção não adequada, as taxas apresentaram tendência ao aumento, com tendência um pouco menor nos municípios com PSF adequado ${ }^{3}$.

Ocorreram mudanças positivas na evolução de algumas taxas de internações por condições sensíveis à atenção ambulatorial, podendo estar associadas à oferta de atenção básica através do PSF de qualidade ${ }^{3}$. A magnitude das mudanças, em geral mais significativa nos municípios onde a oferta da atenção básica do PSF é mais adequada, sugere que a ampliação da cobertura do PSF facilitou o acesso da população aos serviços deste nível de atenção, e que ao mesmo tempo a qualificação da atenção recebida contribuiu para a redução das internações pelas causas selecionadas.

É notável o impacto do SUS na condição de saúde da população, seja pelo relevante desempenhado dos serviços de $A P$ no cotidiano, seja pela redução da busca por serviços de atenção secundários e terciários ${ }^{3}$.

Idosos continuam sendo responsáveis pela maior proporção de gastos com ICSAP, por isso, ressalta-se a importância de subsidiar políticas que fortaleçam a atenção primária no Brasil voltada para este público. Assim como, constante monitoramento dos indicadores relacionados às ICSAP, incluindo gastos envolvidos nesses procedimentos ${ }^{23}$.

A implantação da ESF, características da estrutura das unidades básicas de saúde (UBS) e do processo de trabalho das EAB estão associadas à redução das ICSAPs no Brasil e nos estados, mesmo na presença de outros fatores que podem influenciar o risco de internação, como exemplo econômicos, sociais e de acesso aos serviços de saúde ${ }^{30}$. 


\section{CONCLUSÃO}

Conclui-se que de 2008 a 2015 obtivemos redução de $21,9 \%$ na taxa de ICSAP em idosos da AMMOC. As duas maiores taxas foram registradas nos municípios de Água Doce com 29,4 em 2014 e 16,0 em 2011 e em lbicaré com 14,0 interações por 1.000 habitantes em 2014. As principais causas de ICSAP foram doenças pulmonares com $1.740(23,24 \%)$ casos, insuficiência cardíaca com 1.396 $(18,65 \%)$ e gastroenterites infecciosas com $861(11,50 \%)$ internações.

A redução de ICSAP parece demonstrar melhorias nos serviços de saúde de atenção básica. Com isso, este estudo apresentou fundamental importância para a área da saúde, especialmente para enfermagem, pois grande parte dos profissionais poderão atuar na $\mathrm{AB}$, como enfermeiros assistenciais ou gestores.

A pesquisa permitiu visualizar o objetivo fundamental da atenção primária em relação a saúde da população, realizando seu papel principal que é a promoção de saúde e prevenção de doenças, de maneira integrada e multidisciplinar.

Este estudo contribuiu para um diagnóstico da real situação, funcionalidade e eficácia dos serviços prestados nos municípios pesquisados. Tais resultados poderão servir para instituições acadêmicas reforçar a importância do papel da atenção básica, e também para gestores, de maneira que avaliem o andamento e desenvolvimento da saúde do seu município. Também poderá auxiliar na avaliação dos pontos positivos e negativos do serviço, como redução de gastos com futuras hospitalizações que poderiam ou foram evitadas através do serviço de base.

\section{REFERÊNCIAS}

1 Fernandes VBL, Caldeira AP, Faria AA, Rodrigues Neto JF. Internações sensíveis na atenção primária como indicador de avaliação da Estratégia Saúde da Família. Rev. Saúde Pública. 2009 dez; 43(6):928-936.

2 Costa LQ, Pinto Junior EP, Silva, MGC da. Tendência temporal das Internações por Condições Sensíveis à Atenção Primária em crianças menores de cinco anos de idade no Ceará, 2000 a 2012. Epidemiol. Serv. Saúde. 2017 mar; 26(1):51-60.

3 Elias E, Magajewski F. A Atenção Primária à Saúde no sul de Santa Catarina: uma análise das internações por condições sensíveis à atenção ambulatorial, no período de 1999 a 2004. Rev. Bras. Epidemiol. 2008 dez; 11(4):633-647.

4 Pinto Junior EP, Aquino R, Medina MG, Silva MGC. Efeito da Estratégia Saúde da Família nas internações por condições sensíveis à atenção primária em menores de um ano na Bahia, Brasil. Cad. Saúde Pública. 2018; fev:34(2):2-11. 
5 Magalhães ALA, Morais Neto, OL. Desigualdades intraurbanas de taxas de internações por condições sensíveis à atenção primária na região central do Brasil. Ciênc. Saúde Colet. 2017 jun;22(6):2049-2062.

6 Nedel FB, Facchini LA, Bastos JL, Martín-Mateo M, et al. Conceptual and methodological aspects in the study of hospitalizations for ambulatory care sensitive conditions. Ciênc. Saúde Colet. 2011; set;16(1):1145-1154.

7 Torres RL, Ciosak SI. Panorama das Internações por Condições Sensíveis à Atenção Primária no município de Cotia. Rev. Esc. Enferm. USP. 2014 agos;48(spe):137-144.

8 Romero D, Marques A, Rodrigues JM, Almeida V, Arizaleta CM. A Atenção Primária evita agravos de saúde dos idosos no Brasil? Utilidade do Indicador de Internações por Condições Sensíveis à Atenção Primária (ICSAP) na análise da tendência e condições de vida da população idosa de Rio de Janeiro e Minas Gerais. Anais do XIX Encontro Nacional de Estudos Populacionais. São Pedro/SP. 2014; nov: 1-22.

9 Marques AP, Montilla DER, Almeida WS, Andrade CLT. Internação de idosos por condições sensíveis à atenção primária à saúde. Rev. Saúde Pública. 2014; oct:48(5): 817-826.

10 Brasil. Ministério da Saúde. Sistema de Informação Hospitalar do SUS.

DATASUS Tecnologia da Informação a Serviço do SUS. Morbidade Hospitalar do SUS. 2017. [internet]. Disponível em: http://tabnet.datasus.gov.br/cgi/ tabcgi.exe?sih/cnv/niuf.def.

11 Ammoc. Associação dos Municípios do Meio Oeste de Santa Catarina. Estatuto AMMOC. 2018. [Internet]. Disponível em: https://www.ammoc.org.br/cms/pagina/ver/codMapaltem/112880.

12 Santa Catarina. Secretaria de Estado da Saúde. Internações por Condições Sensíveis à Atenção Primária - ICSAP (Taxa por 10.000 habitantes e Percentual sobre o Total). TABNET/DATASUS. 2013. [Internet]. Disponível em: http://200.19.222.8/cgi/tabcgi.exe?Ind_ICSAP/ICSAP.def.

13 Brasil. Ministério da Saúde - Secretaria de Atenção à Saúde. Portaria Nº 221 de 17 de abril de 2008. Lista Brasileira de Internações por Condições Sensíveis à Atenção Primária. 2008. [Internet]. Disponível em: http://bvsms.saude.gov.br/bvs/saudelegis/sas/2008/prt0221_17_04_2008.html.

14 Previato GF, Nogueira IS, Acorsi C, Baldissera VDA, Mathias TAF. Diminuição de internações por condições sensíveis à Atenção Primária em idosos no estado do Paraná. Rev. Saúde Pública do Paraná. 2017; dez: 18(2):15-24.

15 Ibge - Instituto Brasileiro de Geografia e Estatística. IDH - Índice de Desenvolvimento Humano, Santa Catarina. 2010. Disponível em: https://cidades.ibge.gov.br/brasil/sc/pesquisa/37/30255. 
16 Dourado I, Oliveira VB, Aquino R, Bonolo P, Lima-Costa MF, et al. Trends in Primary Health Care-sensitive Conditions in Brazil The Role of the Family Health Program (Project ICSAP - Brazil). Med Care. 2011 jun; 49(6):577-584.

17 Santos VCF, Ruiz ENF, Roese A, Kalsing A, Gerhardt TE. Internações por condições sensíveis a atenção primária (ICSAP): discutindo limites à utilização deste indicador na avaliação da Atenção Básica em Saúde. R. Eletr. de Com. Inf. Inov. Saúde. 2013 jun;7(2):1-16.

18 Rehem TCMSB, Egry EY. Internações por condições sensíveis à atenção primária no estado de São Paulo. Ciênc. Saúde Colet. 2011; dez:16(12):47554766.

19 Mendonça CS, Harzheim E, Duncan BB, Nunes LN, Leyh W. Trends in hospitalizations for primary care sensitive conditions following the implementation of Family Health Teams in Belo Horizonte, Brazil. Health Policy Plan. 2012; jul;27(4):348-55.

20 Sousa NP, Rehem TCMSB, Santos WS, Santos CE. Internações sensíveis à atenção primária à saúde em hospital regional do Distrito Federal. Rev. Bras. Enferm. 2016 jan-fev;69(1):118-125.

21 Eliopoulos C. Enfermagem Gerontológica. $5^{\mathrm{a}}$ edição. Porto Alegre: Artmed Editora; 2005. p. 247-256.

22 Francisco PMSB, Donalisio MRC, Lattorre MRDO. Tendência da mortalidade por doenças respiratórias em idosos do Estado de São Paulo, 1980 a 1998. Rev. Saúde Pública. 2003 abr; 37(2):191-196.

23 Souza DK, Peixoto SV. Estudo descritivo da evolução dos gastos com internações hospitalares por condições sensíveis à atenção primária no Brasil, 2000-2013. Epidemiol. Serv. Saúde. 2017 jun;26(2):285-294.

24 Gott M, Barnes S, Parker C, Payne S, Seamark D, Gariballa S, et al. Predictors of the quality of life of older people with heart failure recruited from primary care. Age Ageing. 2006 mar; 35(2):172-177.

25 Lloyd-Jones D, Adams RJ, Brown TM, Carnethon M, Dai S, De Simone G, et al. Heart diseade and stroke statistics - 2010 update: a report from the American Heart Association. Circulation, 2010; feb;121(7):948-954.

26 Organización Mundial de La Salud (OMS). Adherencia a los tratamientos a largo plazo: pruebas para la acción. Washington: OMS; 2004.

27 Abete P, Testa L, Della-Morte D, Gargiulo L, Galizia L, de Santis D, et al. Treatment for chronic heart failure in the elderly: current practice and problems. Heart Fail Rev. 2013 jul;18(4):529-551.

28 Filho HMT. Gastroenterites infecciosas. J. Bras. Med. 2013 mar-abr; 101(2):2529. 
29 Tate JE, Burton AH, Boschi-Pinto C, Parashar UD. Global, Regional, and National Estimates of Rotavirus Mortality in Children. Clin. Infect. Dis. 2016; abr; 62(2):96105.

30 Resende APGL, Barbieri AR. Internações por condições sensíveis à atenção primária à saúde decorrentes das doenças cardiovasculares. Texto Contexto Enferm. 2017; agos:26(3):1-8.

\section{AGRADECIMENTOS}

Agradecemos ao curso de Enfermagem, ao Programa de Mestrado em Biociências em Saúde da UNOESC e a Coordenação de Aperfeiçoamento de Pessoal de Nível Superior (CAPES/PNPD) pela oportunidade e incentivo financeiro recebidos.

Artigo recebido em: 23/04/2019

Artigo aprovado em: 16/12/2019

Artigo publicado em: 08/01/2020 